\title{
CONCENTRATIONS OF CYSTEINYL LEUKOTRIENES IN VARIOUS BIOLOGICAL FLUIDS OF CHILDREN WITH BRONCHIAL ASTHMA, ATOPIC DERMATITIS AND FOOD PROTEIN INDUCED ENTEROCOLITIS
}

\author{
Halyna Haiduchyk \\ Department of nutrition and somatic diseases of young children \\ State Institution "Institute Pediatrics, Obstetrics and Gynecology \\ named after academician O. Lukyanova of NAMS of Ukraine” \\ 8 Platona Mayborody str., Kyiv, Ukraine, 04050 \\ gaiduchikgala@ukr.net \\ Oleg Shadrin \\ Department of nutrition and somatic diseases of young children \\ State Institution "Institute Pediatrics, Obstetrics and Gynecology \\ named after academician O. Lukyanova of NAMS of Ukraine" \\ 8 Platona Mayborody str., Kyiv, Ukraine, 04050
}

\begin{abstract}
Clinical manifestation of food allergy is characterized by polymorphic cutaneous, respiratory and gastrointestinal syndromes. Leukotrienes occupy a key place in the pathogenesis of a wide range of inflammatory diseases, including bronchial asthma, allergic rhinitis, atopic dermatitis, hives, allergic conjunctivitis, atherosclerotic cardio-vascular lesions system, inflammatory bowel disease, multiple sclerosis, cancer, etc. Better understanding of general pathophysiological mechanisms of allergic realization put the focus on the studying of cysteinyl leukotrienes biological effects in infants with atopic dermatitis and food-protein induced enterocolitis important.

Aim. To optimize the diagnosis of allergic lesions of the gastrointestinal tract in children.

Methods. The study was conducted in the allergy center and children clinic of the «Institute Pediatrics, Obstetrics and Gynecology named after academician O. Lukyanova of NAMS of Ukraine». Children were included from September 2017 to June 2018.

We examined 60 patients aged from 3 months to 3 years old, including 22 patients with atopic dermatitis, 18 children with food-protein induced enterocolitis, 8 patients with bronchial asthma in the stage of aggravation and 12 practically healthy children (control group).

Medical examination have been perfomed, general IG E and specific serum IG E were defined by ImmunoCAP (Phadia, 100), as well as concentrations of cysteinyl leukotrienes (LTB4, LTC4, LTE4) in biological liquids (serum, saliva, urine) using immunoenzyme method using the production sets of the "Enzolifescience" (USA) company on the analyzer-photometer Multiskan Plus "Labsystems". The results of the received data were processed statistically. The probability of differences was estimated with Student's t-test and Tau Kendall rank correlation test. The difference was considered significant at $\mathrm{p}<0.05$.

Results. A significant increase in the concentrations of cysteinyl leukotrienes (C4, D4, E4) in the blood, urine and saliva was found in infants with allergic skin diseases, gastro-intestinal and respiratory tract surveyed during manifestation the disease compared with the control group.

Comparison of concentrations of leukotrienes in urine and saliva of children with atopic dermatitis (AD), food-protein induced enterocolitis (FPIE) and asthma did not find credible. However, in the serum of patients with asthma, the concentration of cysteinyl leukotrienes was significantly higher $(703.9 \pm 68.7) \mathrm{pg} / \mathrm{ml}$ than in children with enterocolitis induced by dietary proteins $(509.3 \pm 57.4) \mathrm{pg} / \mathrm{ml}$ and significantly did not differ from patients with atopic dermatitis $(695.2 \pm 46.3) \mathrm{pg} / \mathrm{ml}$.

According to the results of Kendall Tau correlation test, no significant Spearman rank correlation was found between the cysteinyl leukotrienes concentration in blood and urine $-r=0.14(p>0.05)$, blood and saliva $r=0.07(p>0.05)$, urine and saliva $r=-0.52(p>0.05)$.

Conclusions. Increase in cysteinyl leukotrienes concentrations in serum, urine and saliva of children of early age with allergic skin diseases, respiratory and gastrointestinal tract was found. The absence of significant Spearman rank correlation between concentrations of leukotrienes in blood and urine, blood and saliva, saliva and urine shows that it is possible to select any biological fluid, saliva or urine, as a non-invasive way to determine the leukotriene concentrations for monitoring activity of allergic inflammation.
\end{abstract}

Keywords: cysteinyl leukotrienes, children of early age, atopic dermatitis, food-protein induced enterocolitis, bronchial asthma.

DOI: $10.21303 / 2504-5679.2019 .00945$ 


\section{Introduction}

In the last decade the rapid growth of allergic pathologies was noted around the world, which the manifestation of is often happens in early childhood as reactions to food [1, 2]. Clinical manifestation of food allergy (FA) is characterized by polymorphic cutaneous, respiratory and gastrointestinal syndromes $[3,4] .50-70 \%$ of children are diagnosed with atopic dermatitis (AD) during the first years of life [5].

Gastrointestinal tract (GI) is directly involved in contact with allergens. Allergic inflammation could be develop in various areas of GI tract, so gastrointestinal form takes the second place in the overall structure of the clinical manifestations of the food allergy $(48-67 \%)[6,7]$.

Allergic inflammation is a complex cascade reaction involving various inflammatory mediators produced by inflammatory effector cells [8].

Along with the leading role of histamine in the development of allergic diseases, lipid mediators leukotrienes (LT) also take an extremely important place in early and late stages of allergic reactions $[9,10]$.

LT occupy a key place in the pathogenesis of a wide range of inflammatory diseases, including bronchial asthma (BA), allergic rhinitis, atopic dermatitis, hives, allergic conjunctivitis, atherosclerotic cardio-vascular lesions system, inflammatory bowel disease, multiple sclerosis, cancer, etc. $[11,12]$.

For the first time cysteinyl LT (Cys-LT) were isolated from leukocytes and characterized by conjugated trien structure. LT are revealed to be organic compounds of the highly active substances group, formed from arachidonic acid (AA), by chipping from the cytoplasmic membrane phospholipids with the participation of the enzyme phospholipase A2.

The transformation of free AA can be accessed in two ways: cyclo-oxigenases AK metabolism pathway involving the formation of prostaglandins and thromboxane A2 and lipooksigenaze with LT release. Free AA first turns into unstable LTA4, then at LTB4 or LTC4, where upon LTC4 LTD4 and LTE4 transforms into LTD4 and LTE4 [13, 14].

Due to the presence of the aminoacid cysteine LTC4, D4 and E4 were called cysteinyl, previously called "slow reacting substance of anaphylaxis" (slow reacting substance), as their release leads to a slowly growing, but prolonged reduction of smooth muscles contractions in bronchi GI tract.

To date, the role of lipid mediators Cys-LT in the pathophysiology of BA was convincingly proved $[15,16]$. Cys-LT have a greater effect on the smooth muscles of respiratory tract compared with the histamine and cause effects typical for BA: an increase of microvascular permeability, the edema formation, an attraction of eosinophils in airway and bronchial infiltration endothelial damage leading to broncho-obstruction $[17,18]$.

Better understanding of general pathophysiological mechanisms of allergic realization put the focus on the studying of Cys-LT biological effects in infants with AD and food-protein induced enterocolitis (FPIE) which is very important.

\section{Aim of the research}

To optimize the diagnosis of allergic lesions of the gastrointestinal tract in children.

\section{Material and methods of the research}

\section{1. Study population}

The study was conducted in the allergy center and children's clinics of the «Institute Pediatrics, Obstetrics and Gynecology named after academician O. Lukyanova of NAMS of Ukraine». Children were included from September 2017 to June 2018.

We examined 60 infants (from 3 months to 3 years): 22 patients with AD, 18 patients with food-protein induced enterocolitis (FPIE) and 12 healthy children (the control group).

Respecting the pathophysiological significance of Cys-LT in children with BA for an objective analysis, we examined Cys-LT concentrations in 8 children of similar age with BA exacerbation (5 patients with persistent atopic BA and 3 children with the virus-induced BA).

Parents of all participants provided informed written consent to clinical investigations. The study design and the procedures were approved by ethical committee (No. 12, 4.09.2017). 


\section{2. IgE assays}

Sensibilization to determine total IgE antibodies and specific IgE antibodies to food and other allergens by ImmunoCAP (Phadia, 100) were done. Results equal to or exceeding $0.35 \mathrm{kUa} / 1$ were considered positive.

\section{3. Cysteinyl leukotrienes}

For determination of the concentrations of Cys-LT (LTB4, LTC4, LTE4) in biological fluids (blood serum, saliva, urine), immunoassay method of «EnzoLifeScience» (United States) company and the analyzer-photometer «Multiskan Plus» Labsystems were used.

\section{4. Statistical analysis}

Mathematical analysis and statistical data processing was carried out on the basis of the license of the software package Statistic for Windows 6.0.RU (StatSoft, United States No. AXXR712D833214FAN5). The likelihood of differences was assessed using the Student's t-test and Kendall Tau rank correlation analysis. P value $<0.05$ was considered statistically significant.

\section{Results}

\section{1. Study population and clinical parameters}

Children were distributed by age and sex. The number of boys and girls was the same (50.0\% and $50.0 \%$, respectively). The average age of surveyed children was (16.5 2.3$)$ months.

According to the analysis, risk factors for FPIE and AD in infants included complications in obstetric history of the mother; the presence of allergies in family anamnesis, especially in mothers, and early artificial feeding.

The first 3 months of life were found as a typical age for onset of the gastrointestinal manifestations of food allergy. Variable clinical picture included refusal of food, intense colic, regurgitation or vomiting, diarrhea with lots of glassy mucus admixture of blood, progressing in permanent presence of the causative food allergens.

Clinical manifestations of AD while the first months of life were associated with cow's milk formula feeding in artificially fed babies and with presence of food with high allergenic potential in mother' ratio in breastfed children.

AD was characterized by chronic recurrent course with a predominance of erythematous-squvamous moderate and severe forms (dryness, peeling, erythema, excoriations) and was accompanied by pronounced itching. The average severity of AD was evaluated as (75.35 \pm 3.20$)$ according to SCORAD score.

The average total $\operatorname{IgE}$ in children was identified and made (136.9 \pm 43.6$) \mathrm{kUA} / \mathrm{l}$ in infants with FPIE and (198.08 \pm 85.6$) \mathrm{kUA} / 1$ (norm $<45 \mathrm{kUA} / \mathrm{l})$ in a group of children with AD. Among food allergens in children with FPIE sensibilization was found to cow's milk proteins in 12 (66.7 \%), egg protein in $10(55.5 \%)$, wheat allergen in $7(38.8 \%)$ and soy protein for $3(16.6 \%)$ children.

Also sensibilization to house dust mites in $2(11.1 \%)$ children and epidermal sensibilization (cat and dogs dander) in 7 (38.8\%) babies were found.

Children with AD were revealed to have sensibilization to food allergens to cow's milk proteins in 10 subjects $(45.4 \%)$, to egg protein in $12(54.5 \%)$ and to wheat flour allergens in $5(22.7 \%)$ persons.

Often polyvalent sensibilization (for more than two allergens) in infants, requires a individual approach to eliminating diet and prevention of «Atopic March».

The paraclinical data analysis identified changes in blood as hypochromic anemia and eosinophilia in 12 (30.0\%) children, with average eosinofils level (8.4 $\pm 1.6 \%)$ in both groups.

Parameters of the inflammatory process were determined in studied concentration of Cys-LT (C4, D4, E4) formed as a result of lipo-oxigenic pathway of arachidonic acid metabolism.

Analysis of the received results revealed a significant increase in the concentrations of Cys-LT (C4, D4, E4) in blood, urine, saliva of children of early age with AD, FPIE and BA surveyed during of the disease manifestation, compared with the control group (Table 1). 
Table 1

Concentration of Cys-LT (LTB4, LTC4, LTE4) in biological fluid (blood, urine, saliva) of participants, $\mathrm{pg} / \mathrm{ml}(\mathrm{M} \pm \mathrm{m})$

\begin{tabular}{ccccc}
\hline \multirow{2}{*}{ Biological fluids } & \multicolumn{4}{c}{ Data in studied groups $(\mathbf{n}=\mathbf{6 0})$} \\
\cline { 2 - 5 } & Control group $(\mathbf{n}=\mathbf{1 2})$ & BA (n=8) & FPIE (n=18) & AD (n=22) \\
\hline Blood, pg/ml & $(90.6 \pm 16.8)$ & $(703.9 \pm 68.7)^{* \wedge}$ & $(509.3 \pm 57.4)^{* \wedge}$ & $(695.2 \pm 46.3)^{* \wedge}$ \\
Urine, $\mathrm{pg} / \mathrm{ml}$ & $(78.2 \pm 24.7)$ & $(424.0 \pm 115.6)^{*}$ & $(338.5 \pm 14.5)^{*}$ & $(308.2 \pm 34.2)^{*}$ \\
Saliva, $\mathrm{pg} / \mathrm{ml}$ & $(55.5 \pm 26.2)$ & $(461.3 \pm 107.0)^{*}$ & $(239.7 \pm 19.3)^{*}$ & $(265.3 \pm 25.2)^{*}$
\end{tabular}

Note: * - Significant difference with control ( $p<0.05) ;{ }^{\wedge}$ - significant difference in BA, FPIE, AD groups $(p<0.05)$

Comparison of concentrations of Cys-LT in urine and saliva of children with FPIE, BA and AD did not show significant difference. Serum concentration of Cys-LT in patients with asthma was significantly higher (703.9 \pm 68.7$) \mathrm{pg} / \mathrm{ml}$ than in children with the FPIE $(509.3 \pm 57.4) \mathrm{pg} / \mathrm{ml}$ and reliably did not differ from that of patients with AD $(695.2 \pm 46.3) \mathrm{pg} / \mathrm{ml}$.

Children with FPIE had serum Cys-LT concentration $(509.3 \pm 57.4) \mathrm{pg} / \mathrm{mL}$ was significantly higher than in saliva $(239.7 \pm 19.3) \mathrm{pg} / \mathrm{ml}$ and urine $(338.5 \pm 14.5) \mathrm{pg} / \mathrm{ml}$, and Cys-LT content in the urine was higher than in saliva. Similar characteristics were identified for children with AD where concentrations of Cys-LT in the blood $(695.2 \pm 46.3) \mathrm{pg} / \mathrm{mL}$ were significantly higher than in saliva $(265.3 \pm 25.2) \mathrm{pg} / \mathrm{ml}$ and urine $(308.2 \pm 34.2) \mathrm{pg} / \mathrm{ml}$, but Cys-LT concentration in urine and saliva were not significantly different.

Kendall Tau rank correlation analysis was provided to determine the relationship between Cys-LT concentration in the blood, urine and saliva of surveyed children. According to the results, correlation analysis revealed no significant Spearman rank correlation between concentrations of Cys-LT in blood and urine $-r=0.14(p>0.05)$, blood and saliva $r=0.07(p>0.05)$, urine and saliva $\mathrm{r}=-0.52(\mathrm{p}>0.05)$.

Results may suggest the possibility of choosing any biological fluid, saliva or urine in particular, as non-invasive method to determine the concentrations of Cys-LT and to monitor allergic inflammation activity.

Implementation of the biological effects of Cys-LT includes interaction with specific receptors-Cys-and Cys-LT1 LT2, so blocking synthesis and reception LT and, consequently, reduce their physiological effects by consumption of antihistamines and glucocorticosteroids is not justified. In this regard, use of drugs selectively blocking the Cys-LT receptors seems to be pathophysiologically justified.

\section{Discussion}

Nowadays recent study of lipid mediators (cysteinyl leukotrienes) participation in the pathophysiology of allergic inflammation with skin and gastrointestinal tract clinical manifestation in infants is relevant regarding the possibility of their possible pharmacological correction.

There is evidence of an increase in LT production in the pathogenesis of AD [19]. The levels of urinary LTE4 and serum eosinophilic cationic protein (ECP) in children with AD are higher than in children without allergic diseases; there was a significant correlation between LTE4 in urine and ECP in serum and the SCORAD index [19]. CysLT levels were also investigated during biopsy of the esophageal mucosa in children with eosinophilic esophagitis and in normal children. CysLT levels in patients with eosinophilic esophagitis are similar to those in the control and do not depend on the severity of inflammation. Although this is contrary to the use of Cys-LT antagonists in the treatment of eosinophilic esophagitis [20].

The obtained data about high concentrations of Cys-LT in various biological substances (blood, urine, saliva) is an objective criteria of eosinophils participation in the development of clinical symptoms of AD and FPIE and may enhance understanding of pathogenic opportunities in influence the course of allergic inflammation in infants with gastrointestinal tract and skin allergy. 
Cys-LT concentration measured in biological fluids (blood, urine, saliva) is suggested to be an effective and non-invasive method to control an allergic process activity.

A significant increase in the cysteinyl leukotrienes concentration in various biological fluids indicates the prospect of studying the effectiveness of cisteinyl leukotrienes receptor inhibitors for treatment of children with AD and the FPIE.

Obtained results confirm the importance of lipid mediators (leukotrienes, cysteinyl) in the pathophysiology of atopic dermatitis and food-protein induced enterocolitis in infants. A promising direction for further research is to study the effectiveness of leukotriene cysteinyl receptors blockers (antileukotriene drugs) in infants with allergic skin and gastrointestinal tract.

\section{Conclusions}

1. We discovered high concentration of cysteinyl leukotrienes (LTB4, LTC4, LTE4) in biological fluids (blood serum, urine, saliva) in infants with atopic dermatitis, and food-protein induced enterocolitis compared to the control group.

2. The absence of significant rank correlation between concentrations of leukotrienes in blood and urine, blood and saliva, saliva and urine shows the possiblity to select any biological fluid, saliva or urine in particular, as a non-invasive method for determination of the cysteinyl leukotrienes concentrations for monitoring of allergic inflammation activity.

\section{References}

[1] Koletzko, S., Niggemann, B., Arato, A., Dias, J. A., Heuschkel, R., Husby, S. et. al. (2012). Diagnostic Approach and Management of Cow's-Milk Protein Allergy in Infants and Children. Journal of Pediatric Gastroenterology and Nutrition, 55 (2), 221-229. doi: http://doi.org/10.1097/mpg.0b013e31825c9482

[2] Berin, M. C., Sampson, H. A. (2013). Food allergy: an enigmatic epidemic. Trends in Immunology, 34 (8), 390-397. doi: http:// doi.org/10.1016/j.it.2013.04.003

[3] Maloney, J., Nowak -Wegrzyn, A. (2014). Educational clinical case series for pediatric allergy and immunology: Allergic proctocolitis, food protein-induced enterocolitis syndrome and allergic eosinophilic gastroenteritis with protein-losing gastroenteropathy as manifestations of non-IgE-mediated cow's milk allergy. Journal of Pediatric Allergy and Immunology, 18 (4), 360-367. doi: http://doi.org/10.1111/j.1399-3038.2007.00561.x

[4] Waserman, S., Bégin, P., Watson, W. (2018). IgE-mediated food allergy. Allergy, Asthma \& Clinical Immunology, 14 (S2). doi: http://doi.org/10.1186/s13223-018-0284-3

[5] Macharadze, D. (2013). Clinical features of food allergy in children. Pediatrics, 92 (3), 110-116. Available at: https://pediatriajournal.ru/files/upload/mags/329/2013_3_3714.pdf

[6] Nowak-Węgrzyn, A., Chehade, M., Groetch, M. E., Spergel, J. M., Wood, R. A., Allen, K. et. al. (2017). International consensus guidelines for the diagnosis and management of food protein-induced enterocolitis syndrome: Executive summary-Workgroup Report of the Adverse Reactions to Foods Committee, American Academy of Allergy, Asthma \& Immunology. Journal of Allergy and Clinical Immunology, 139 (4), 1111-1126. doi: http://doi.org/10.1016/j.jaci. 2016.12.966

[7] Sicherer, S. (2013). Clinical aspects of gastrointestinal food allergy in childhood. Pediatrics, 111 (6), 1609-1616.

[8] Boyce, J. A. (2007). Mast cells and eicosanoid mediators: a system of reciprocal paracrine and autocrine regulation. Immunological Reviews, 217 (1), 168-185. doi: http://doi.org/10.1111/j.1600-065x.2007.00512.x

[9] Pelz, B. J., Wechsler, J. B., Amsden, K., Johnson, K., Singh, A. M., Wershil, B. K. et. al. (2016). IgE-associated food allergy alters the presentation of paediatric eosinophilic esophagitis. Clinical \& Experimental Allergy, 46 (11), 1431-1440. doi: http:// doi.org/10.1111/cea.12776

[10] El-Ghoneimy, D., El-Sayed, Z. (2014). Leukotrienes and leukotriene modifiers in pediatric allergic diseases. Egyptian Journal of Pediatric Allergy and Immunology, 12 (1), 3-12. Available at: http://www.jespai.org/cms/upload/1395779928.pdf

[11] Papadopoulos, N. G., Arakawa, H., Carlsen, K.-H., Custovic, A., Gern, J., Lemanske, R. et. al. (2012). International consensus on (ICON) pediatric asthma. Allergy, 67 (8), 976-997. doi: http://doi.org/10.1111/j.1398-9995.2012.02865.x

[12] Rothenberg, M. E. (2004). Eosinophilic gastrointestinal disorders (EGID). Journal of Allergy and Clinical Immunology, 113 (1), 11-28. doi: http://doi.org/10.1016/j.jaci.2003.10.047

[13] Galli, S. J., Grimbaldeston, M., Tsai, M. (2008). Immunomodulatory mast cells: negative, as well as positive, regulators of immunity. Nature Reviews Immunology, 8 (6), 478-486. doi: http://doi.org/10.1038/nri2327 
[14] Meyer, R., Fleming, C., Dominguez-Ortega, G., Lindley, K., Michaelis, L., Thapar, N. et. al. (2013). Manifestations of food protein induced gastrointestinal allergies presenting to a single tertiary paediatric gastroenterology unit. World Allergy Organization Journal, 6, 13. doi: http://doi.org/10.1186/1939-4551-6-13

[15] Global strategy for asthma management and prevention (2015). National institutes of health. National Heart, lung and Blood Institute. Available at: http://www.ginasthma.org

[16] Scaparrotta, A., Di Pillo, S., Attanasi, M., Rapino, D., Cingolani, A., Consilvio, N. P. et. al. (2012). Montelukast versus inhaled corticosteroids in the management of pediatric mild persistent asthma. Multidisciplinary Respiratory Medicine, 7 (1), 1-9. doi: http://doi.org/10.1186/2049-6958-7-13

[17] Buck, M. (2015). Montelukast: a review of recent studies in pediatric asthma and allergic rhinitis. Pediatric Pharmacology, 21 (4). Available at: http://www.medscape.com/viewarticle/845468

[18] Pei, A. Y. S., Chan, H. H. L., Leung, T. F. (2001). Montelukast in the treatment of children with moderate-to-severe atopic dermatitis: A pilot study. Pediatric Allergy and Immunology, 12 (3), 154-158. doi: http://doi.org/10.1034/j.1399-3038.2001.012003154.x

[19] Oh, J., Lee, H. (2004). Study of urinary leukotriene E4 and serum eosinophil cationic protein in children with atopic dermatitis and their correlation with disease activity. Journal of Allergy and Clinical Immunology, 113 (2), S56. doi: http:// doi.org/10.1016/j.jaci.2003.12.165

[20] Gupta, S. K., Peters-Golden, M., Fitzgerald, J. F., Croffie, J. M., Pfefferkorn, M. D., Molleston, J. P. et. al. (2006). Cysteinyl Leukotriene Levels in Esophageal Mucosal Biopsies of Children with Eosinophilic Inflammation: Are They All the Same? The American Journal of Gastroenterology, 101 (5), 1125-1128. doi: http://doi.org/10.1111/j.1572-0241.2006.00557.x

\title{
PECULIARITIES OF TLR-2 (ARG753GLN) AND TLR-4 (ASP299GLY) POLYMORPHISM PREVALENCE IN PATIENTS WITH ACUTE BRUCELLEIS AND CARDIOVASCULAR SYSTEM DISEASES
}

\author{
Elchin Huseynov Mammad oglu \\ Department of Infectious Diseases \\ Azerbaijan Medical University \\ 23 Bakikhanov str., Baku, Azerbaijan, AZ1022 \\ elchinhuseynov@mail.ru
}

\begin{abstract}
Currently, there are single data on the relationship between TLR-2 polymorphisms (Arg753Gln) and TLR-4 (Asp299Gly) polymorphisms and susceptibility to brucellosis. Therefore, the aim of the study was to determine the frequency of TLR-2 (Arg753Gln) and TLR-4 (Asp299Gly) polymorphisms in patients with acute brucellosis with cardiovascular lesions in the Republic of Azerbaijan.

Materials and methods: 178 patients with a brucellosis clinic were examined. According to the criteria for inclusion in the study, only 120 people fully met all the criteria, which made up the main group. The control group consisted of 30 healthy individuals. TLR-2 (Arg753Gln) and TLR-4 (Asp299Gly) polymorphisms were also determined for all patients in both groups. In order to assess the state of the cardiovascular system, an electrocardiogram was recorded, blood pressure was measured, and an ultrasound scan of the heart was performed for all patients.

Results: it was found that 93 patients $(77.50 \pm 3.13 \%)$ with acute brucellosis had some or other signs of impairment in the work of the cardiovascular system, identified clinically or as a result of functional examination. Among carriers of the Asp/Gly genotype of the TLR-4 gene, an increased risk of brucellosis with CVS diseases was determined $\left(\chi^{2}=30.19 ; \mathrm{p}<0.0001 ; \mathrm{OR}=24.29 ; 95 \% \mathrm{CI}[5.45-\right.$ 108.37]), while the carriage of the homozygous genotype Asp/Asp, by contrast, had a protective effect on the development of brucellosis $(\mathrm{OR}=0.06,95 \% \mathrm{CI}[0.02-0.20])$. Among the carriers of the Arg/Gln genotype and the Gln/Gln genotype of the TLR-2 gene, a significantly increased risk of brucellosis with CVS diseases was determined $\left(\chi^{2}=5.68 ; \mathrm{p}=0.02 ; \mathrm{OR}=3.10 ; 95 \% \mathrm{CI}[0.99-9.67]\right)$ and $(\mathrm{OR}=2.48$;
\end{abstract}

